\title{
Chiral lattice gauge theories from warped domain walls and Ginsparg-Wilson fermions
}

\section{Tanmoy Bhattacharya*}

T-8, MS B285, Los Alamos National Laboratoy, Los Alamos, NM 87545, USA

E-mail: tanmoyalanl.gov

\section{Matthew R. Martin}

OTIP, University of Chicago, 5555 S. Woodlawn Ave., Suite 300, Chicago, IL 60637, USA

E-mail: m-martin@uctech.uchicago.edu

\section{Erich Poppitz}

Department of Physics, University of Toronto, Toronto, ON M5S 1A7, Canada

poppitz@physics.utoronto.ca

\begin{abstract}
We propose a construction of a 2-dimensional lattice chiral gauge theory. The construction may be viewed as a particular limit of an infinite warped 3-dimensional theory. We also present a "single-site" construction using Ginsparg-Wilson fermions which may avoid, in both 2 and 4 dimensions, the problems of waveguide-Yukawa models.
\end{abstract}

XXIVth International Symposium on Lattice Field Theory

July 23-28, 2006

Tucson, Arizona, USA

${ }^{*}$ Speaker. 


\section{Introduction and summary}

It is well known that the Nielsen-Ninomiya no-go theorem [1] forbids discretizing chiral gauge theories on the lattice maintaining all their symmetries [2,3] because of 'doubler' modes of same charge and opposite chirality appearing in the physical spectrum. Removing these requires sacrificing the unbroken gauge symmetry which then appears only at the continuum limit [4]. Recently, a proposal $[5,6]$ was made to implement this as a limit of a spontaneously broken higher dimensional gauge theory in a curved background field. Apart from the curvature that allows the decoupling of the gauge and fermion masses from each other, this is similar to the 'wave-guide model' [7, 8], which is known not to give a chiral theory[9]. Unfortunately, the Goldstone mode that forms part of the gauge boson in the unitary gauge remains strongly coupled and the model cannot be analyzed without numerical simulations. In this talk, we first consider an analogous construction in 3-dimensional Anti-de Sitter space in an attempt to construct a 2-dimensional chiral gauge theory, and show that the Goldstone mode remains perturbative. We then propose a related, simplified 'one-site' model which consists of only a 2-dimensional lattice theory where massless fermions are introduced using the Ginsparg-Wilson mechanism [10] for imposing a modified chiral symmetry. The symmetries and anomalous Ward identities in this model are as expected in a chiral gauge theory. More details about the construction of the models can be found in [11].

\section{Domain Wall Fermions in Two Dimensions}

We work with light-cone coordinates:

$$
x^{ \pm} \equiv t \pm x \quad \rightarrow \quad 2 \partial_{ \pm}=\partial_{t} \pm \partial_{x}
$$

so that the Lagrangian for a charged, massive, Dirac fermion is:

$$
\mathscr{L}=2 i \bar{\psi}_{-}\left(\partial_{+}-i A_{+}\right) \psi_{-}+2 i \bar{\psi}_{+}\left(\partial_{-}-i A_{-}\right) \psi_{+}+m_{D} \bar{\psi}_{+} \psi_{-}+m_{D}^{*} \bar{\psi}_{-} \psi_{+} .
$$

The left handed complex field $\psi_{-}$and the right handed $\psi_{+}$comprise the two dimensional Dirac field. The bar on these one-component fields indicates complex conjugation. The subscript $D$ on the mass indicates that it is a Dirac type mass term: it does not break a gauge symmetry in contrast to masses of the Majorana type: $m_{M} \psi_{+} \psi_{-}+$h.c..

The domain wall fermions [7] arise as edge states of a theory with a long third dimension labeled with the coordinate $z$. Since we will not take the lattice spacing in this extra dimension to zero, we write the theory in the 'deconstruction picture' [12] where the $z$ direction is discrete but the other two form a continuum. To make one of the edge states heavy, we place a charged and a neutral model back to back (Fig. 1), sharing one (the $k^{\text {th }}$ ) edge:

$$
\begin{aligned}
& \sum_{i=1}^{k}\left[2 i \bar{\psi}_{i-} \partial_{+} \psi_{i-}+2 i \bar{\psi}_{i+} \partial_{-} \psi_{i+}+\left(m_{i} \bar{\psi}_{i-} \psi_{i+}+\text { h.c. }\right)\right]+\sum_{i=2}^{k}\left(m_{i}^{\prime} \bar{\psi}_{i+} \psi_{i-1,-}+\text { h.c. }\right) \\
& \quad+\sum_{i=k+1}^{N}\left[2 i \bar{\psi}_{i-}\left(\partial_{+}-i A_{+}\right) \psi_{i-}+2 i \bar{\psi}_{i+}\left(\partial_{-}-i A_{-}\right) \psi_{i+}\right. \\
& \left.+\left(m_{i} \bar{\psi}_{i-} \psi_{i+}+\text { h.c. }\right)\right]+\sum_{i=k+2}^{N}\left(m_{i}^{\prime} \bar{\psi}_{i+} \psi_{i-1,-}+\text { h.c. }\right)
\end{aligned}
$$




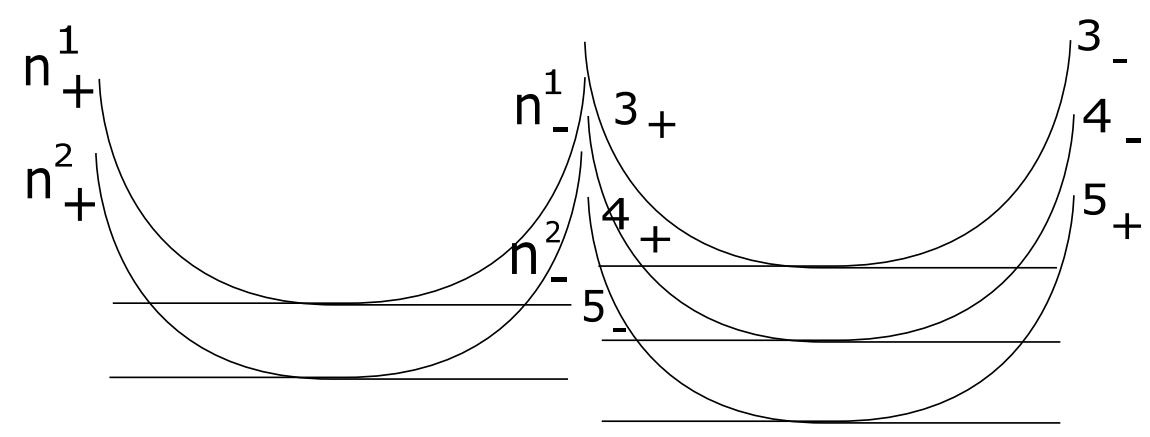

Figure 1: The wave functions of the light modes. The right half is gauged; the left is neutral.

and couple them with a Higgs' field as $y \bar{\psi}_{k+1,+} \phi \psi_{k,-}+$ h.c.. When the gauge field is constant along the $z$ direction, this model is known to be non-chiral [9] since either the gauge boson becomes as heavy as the edge state one is trying to remove, or new massless edge modes appear in the theory. The odd Kaluza-Klein modes of the gauge field, however, are not affected by the Higgs' mechanism, but since the masses for all of them are controlled by the same scale, this does not lead to a two-dimensional theory in the appropriate limit either.

In [5] it was argued that the situation is different [13] when one is considering a non-trivial background metric along the extra dimension. In this case the scaling of the gauge boson mass could be different from that of the fermion mass in the presence of a symmetry breaking vacuum expectation value on the domain wall boundaries. This led to a possibility of recovering a chiral gauge theory in the limit when the warping (the background curvature of the extra dimension) is increased to infinity.

In order for the limit to reproduce a two dimensional gauge theory, we must also need the fundamental scale of the theory, $\Lambda_{\chi G T} \sim g_{2}$, to satisfy $m_{K K} \gg \Lambda_{\chi G T} \gg m_{A_{0}}$, so that we can take the scale of three dimensional physics, $m_{K K}$, to infinity and the mass of lightest gauge boson, $m_{A_{0}}$ to zero and still obtain a non-trivial two dimensional gauge theory. To achieve this, we start with a slice of 3-dimensional Anti-de Sitter space, $A d S_{3}$, with the coordinates given by:

$$
d s^{2}=\left(\frac{R}{z}\right)^{2}\left(\eta_{\mu \nu} d x^{\mu} d x^{v}-d z^{2}\right)
$$

between $z=R$ (the UV 'brane') and $z=R^{\prime} \gg R$ (the IR 'brane'). Contrary to the practice in four dimensions [5, 14], the Higgs mechanism needs to be implemented on the UV brane to achieve the desired separation of scales. ${ }^{1}$ We discretize this space such that the lattice spacing $\delta z_{i}=a z_{i}$, so that a discrete part of the anti-deSitter translation symmetry (away from the boundaries) can be recovered by tuning as the naïve continuum limit is taken in the two flat directions alone.

We now consider the perturbative expansion of the deconstructed Lagrangian in the $A_{z}=0$ gauge:

$$
-\frac{1}{4} \sum_{i=1}^{N} \frac{a z_{i}}{R g_{3}^{2}}\left[F_{\mu \nu}^{i}\right]^{2}+\frac{1}{2} D_{\mu}^{1} \phi^{*} D^{1 \mu} \phi-V(\phi)+\frac{1}{2} \sum_{i=1}^{N-1} \frac{a z_{i}^{2}}{R g_{3}^{2}} \frac{\left(A_{\mu}^{i+1}-A_{\mu}^{i}\right)^{2}}{\left(a z_{i}\right)^{2}}+\ldots
$$

\footnotetext{
${ }^{1}$ In fact, it is easy to see that the tree level spectrum of the entire spin-1 KK tower in $A d S_{3}$ is identical to that in $A d S_{5}$ with reversed boundary conditions; we thank T. Gherghetta for discussions.
} 
$\mu, v$ run over the flat directions, the dots represent interaction terms in non-Abelian theories, the $D^{1}$ is a covariant derivative under the two-dimensional gauge group on the first slice, and we have suppressed coordinates in the $x^{\mu}$ direction. The gauge coupling of each slice gauge group is then given by

$$
\frac{1}{g_{i}^{2}}=\frac{a z_{i}^{2}}{R g_{3}^{2}}
$$

and the low energy gauge coupling, at tree level, is approximately given by

$$
\frac{1}{g_{2}^{2}}=\sum_{i=1}^{N} \frac{1}{g_{i}^{2}}=\frac{R^{\prime 2}}{2 R g_{3}^{2}} .
$$

Thus, our hierarchy of mass scales can be written as $1 \gg a z_{i}^{2} g_{i}^{2} \gg 1 / N a$, and we take the large $N$ limit maintaining $g_{i}^{2} \sim 1 / z_{i}^{2} \sqrt{N}$. The fact that we can take $N$ large and keep the individual gauge couplings in $A d S_{3}$ small is due to its superrenormalizable bulk.

In the fermion sector, we start with two neutral Dirac spinors, $n_{ \pm}^{1}$ and $n_{ \pm}^{2}$, and give them Dirac and Majorana masses to leave chiral Weyl states $n_{ \pm}$near the two boundaries. We allow the Higgs' field on the UV boundary to couple the charged edge state to a neutral state: $y\langle\phi\rangle \bar{l}_{+} n_{-}$, and leave the other charged edge state $l_{-}$and neutral $n_{+}$in the low energy spectrum. The measure of the path integral is completely well defined, and the anomaly appears when the symmetry breaking Majorana mass is generated.

We study the anomaly structure of the ' 345 ' theory containing left-handed fermions of charge 3 and 4 as well as right handed fermions of charge 5 , and call them $3_{-}, 4_{-}$, and $5_{+}$, respectively. Before adding gauge breaking mass terms, our warped domain wall construction necessarily contains the mirror fermions as well, $3_{+} 4_{+}$, and $5_{-}$, as well as two neutral modes to give masses to the mirror fermions. If only Dirac masses are contained in the theory, then one global fermion number $U(1)$ symmetry will remain, and to obtain the 't Hooft operator $\left(3_{-}\right)^{3} \partial_{+}\left(4_{-}\right)^{4}\left(\overline{5}_{+}\right)^{5}$, Majorana masses are needed on the lattice [15, 5].

With all of the masses discussed above, the only remaining exact symmetry in the theory is the global part of the gauge symmetry and the ' 345 ' symmetry. However, the 't Hooft operator preserves also another global symmetry, '133,' where the $4_{-}$and $5_{+}$transform with three times the phase of the $3_{-}$. We speculate that either this 133 symmetry will emerge in the IR, or else we have found a theory which preserves no symmetry beyond the gauged 345, which can happen if 133-violating operators (e.g. four-fermi operators in 2 dimensions) remain relevant in the IR.

There does exist, however, a one-site model using Ginsparg-Wilson (GW) fermions which has precisely the light field spectrum expected of the continuum theory. The advantage of this formulation is that its chiral symmetries are exact symmetries of the discretized theory. A strong-coupling analysis indicates that the spectrum of this theory is chiral and the fermion measure has no phase ambiguity. This theory arises as a "one-site limit" of our construction, using 2-dimensional GW fermions in order to implement exact lattice chiral symmetries that arise for the edge states only in the large $N$ limit. Schematically, the field content and couplings of the model are represented in Fig. 2. There are, for the $345 U(1)$ theory, three 2-dimensional Dirac fermions, $\Psi_{3}, \Psi_{4}, \Psi_{5}$, charged under the $U(1)$ gauge group with charges $3,4,5$, respectively. There is also a neutral Dirac fermion, $\Psi_{0}$. 


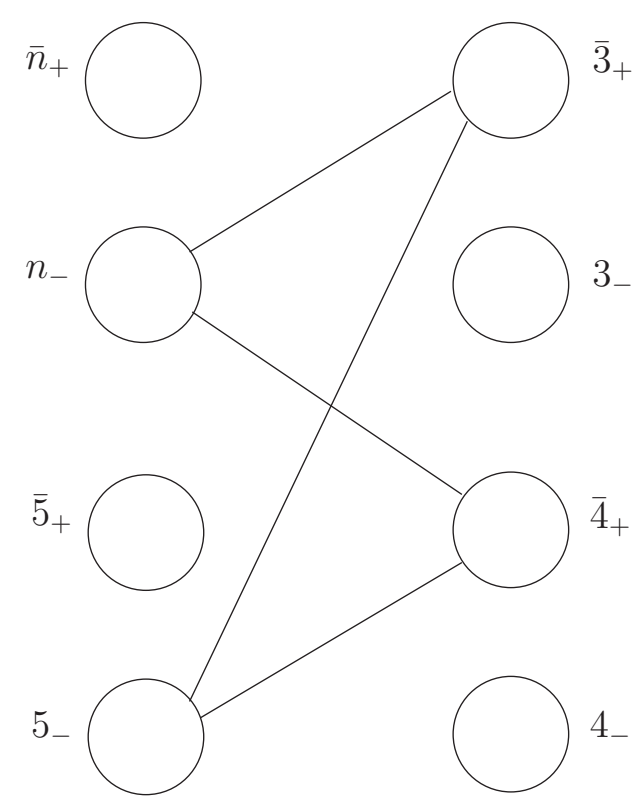

Figure 2: The GW construction of the 345 theory: The lines represent arbitrary $\mathscr{O}(1 / a)$ masses of both Dirac and Majorana type.

There kinetic terms are given by

$$
S_{k i n}=\sum_{q=0,3,4,5} \sum_{x, y} \bar{\Psi}_{q}(x) D_{q}(x, y) \Psi_{q}(y),
$$

where $D_{q}$ is the GW operator for a fermion of charge $q$, obeying the GW relation [3] $\left\{D_{q}, \gamma_{5}\right\}=$ $D_{q} \gamma_{5} D_{q}$. The measure of the path integral is well defined for this vector like theory, but is not invariant under the chiral symmetries rotating each individual $\Psi$ field, and the only chiral symmetries are [16] the 345s, the 133s, and the symmetries rotating the neutral fermions. To construct our candidate ' 345 ' chiral lattice theory, we introduce a unitary higgs field (and ignore ultraviolet difficulties [17]), $\phi(x)$, living on the lattice sites and use $\mathrm{it}^{2}$ to write all possible Dirac and Majorana mass terms ${ }^{3}$ that violate all symmetries (Fig. 2) of the kinetic term except $U(1)_{3,-} \times U(1)_{4,-} \times$ $U(1)_{5,+} \times U(1)_{0,+}$.

To ensure that the dynamics of this theory reproduces that of the desired unbroken chiral gauge theory, we next focus our attention on the coupling of the Higgs field to the fermions, as well as on its kinetic term (i.e., the mass term for the gauge field):

$$
S=S_{W i l s o n}+S_{k i n}+S_{m a s s}+\frac{\kappa}{2} \sum_{x} \sum_{\hat{\mu}}\left[2-\left(\phi(x)^{*} U(x, \hat{\mu}) \phi(x+\hat{\mu})+\text { h.c. }\right)\right],
$$

appropriately modified to restrict the gauge field path integral to admissible gauge field backgrounds [3]. If there the unbroken phase with $\langle\phi\rangle=0$ remains with these Yukawa interactions

\footnotetext{
${ }^{2}$ Use of the GW fermions in these terms makes the theory non-reflection positive, but that is common in theories with massless fermions [18].

${ }^{3}$ With only Dirac masses, there is an extra zero mode at the end of the Brillouin zone and the spectrum is non-chiral.
} 
to non-ultralocal fermions, one expects [19] the fermions to be massive, but the gauge boson to remain massless, the gauge symmetry thus emerging in the infrared by the FNN mechanism [20, 17]. To check whether new light fermion states arise in this phase, we study the weak gauge coupling strong Yukawa coupling limit of this theory. The resulting theory is a unitary Higgs-Yukawa model in the symmetric phase of the lattice $O(2)$ model. Since $d \Psi=d \Psi_{+} d \Psi_{-}$, the lattice partition function factorizes, in a trivial gauge background, into a product $Z=Z_{\text {light }} \times Z_{\text {mirror }}$ :

$$
\begin{aligned}
Z_{\text {light }} & =\int \prod_{x} d \Psi_{3,-} d \Psi_{4,-} d \Psi_{5,+} d \Psi_{0,+} e^{-S_{\text {kin }}\left(\Psi^{\text {light }}\right)} \\
Z_{\text {mirror }} & =\int \prod_{x} d \Psi_{3,+} d \Psi_{4,+} d \Psi_{5,-} d \Psi_{0,-} d \phi \\
\times & e^{-S_{\text {kin }}^{\text {miror }}\left(\Psi^{\text {mirror }}\right)-S_{\kappa}(\phi)-S_{\text {mass }}\left(\Psi^{\text {mirror }}\right)}
\end{aligned}
$$

For conciseness, we omitted the conjugate fields in the measure and denoted collectively by $\Psi_{\text {light }}$ the fields $\Psi_{3,-}, \Psi_{4,-}, \Psi_{5,+}, \Psi_{0,+}$, and by $\Psi_{\text {mirror }}$ the heavy charged mirrors $\Psi_{3,+}, \Psi_{4,+}, \Psi_{5,-}$, and the neutral $\Psi_{0,-}$. Note that it was crucial for this factorization that the kinetic terms split into light and mirror modes in (2.9) exactly:

$$
\bar{\Psi}_{q} D_{q} \Psi_{q}=\bar{\Psi}_{q,+} D_{q} \Psi_{q,+}+\bar{\Psi}_{q,-} D_{q} \Psi_{q,-},
$$

where the cross terms vanish due to the GW relation. In the strong Yukawa limit, the mirror kinetic terms vanish, and the mirror determinant is non-zero. Ideally, turning on a small gauge coupling will not cause a dramatic rearrangement of the spectrum except a mass for the gauge boson will be generated if the theory is anomalous [21]. To argue for this, we note that one expects that all effects of the mirror fermions on the gauge field effective action are local and vanish as the Yukawa coupling becomes infinity.

We should also note that nothing (except for the need, coming from 2 dimensional Lorentz invariance, to introduce the spectator neutral fermions) about the proposal considered in this section is intrinsically 2-dimensional. In fact, all the steps and relevant properties, including the factorization (2.10) of the Ginsparg-Wilson fermion kinetic terms and the existence of a 'high-temperature' disordered phase of the compact Higgs variables, hold in a four-dimensional theory as well, particularly in the abelian case considered here.

\section{Acknowledgments}

We would like to thank Joel Giedt for contributions to the early part of this work and Yuri Shirman for insightful discussions. T.B. and M.M. are supported in part by the U.S. Department of Energy under contract W-7405-ENG-36. E.P. acknowledges support by the National Science and Engineering Research Council of Canada (NSERC).

\section{References}

[1] H. B. Nielsen and M. Ninomiya, Phys. Lett. B 105, 219 (1981); Nucl. Phys. B 193, 173 (1981).

[2] M. Golterman, Nucl. Phys. Proc. Suppl. 94, 189 (2001) [arXiv:hep-lat/0011027]. M. Golterman and Y. Shamir, Phys. Rev. D 70, 094506 (2004) [arXiv:hep-lat/0404011]. 
[3] M. Luscher, arXiv:hep-th/0102028.

[4] P. V. D. Swift, Phys. Lett. B 145, 256 (1984); S. Aoki, I. H. Lee and S. S. Xue, Phys. Lett. B 229, 79 (1989); I. Montvay, Phys. Lett. B 199, 89 (1987); I. Montvay, Phys. Lett. B 205, 315 (1988).

A. Borrelli, L. Maiani, R. Sisto, G. C. Rossi and M. Testa, Nucl. Phys. B 333, 335 (1990);

P. Hernandez and R. Sundrum, Nucl. Phys. B 455, 287 (1995) [arXiv:hep-ph/9506331].

[5] T. Bhattacharya, C. Csaki, M. R. Martin, Y. Shirman and J. Terning, JHEP 0508, 061 (2005) [arXiv:hep-lat/0503011].

[6] T. Bhattacharya, R. Gupta, M. R. Martin, Y. Shirman, C. Csaki and J. Terning, PoS LAT2005, 136 (2005) [arXiv:hep-lat/0510073].

[7] D. B. Kaplan, Phys. Lett. B 288, 342 (1992) [arXiv:hep-lat/9206013].

[8] D. B. Kaplan, Nucl. Phys. Proc. Suppl. 30, 597 (1993).

[9] M. F. L. Golterman, K. Jansen, D. N. Petcher and J. C. Vink, Phys. Rev. D 49, 1606 (1994) [arXiv:hep-lat/9309015]. M. F. L. Golterman and Y. Shamir, Phys. Rev. D 51, 3026 (1995) [arXiv:hep-lat/9409013].

[10] P. H. Ginsparg and K. G. Wilson, Phys. Rev. D 25, 2649 (1982). M. Luscher, Phys. Lett. B 428, 342 (1998) [arXiv:hep-lat/9802011].

[11] T. Bhattacharya, M. R. Martin, and E. Poppitz, [arXiv:hep-lat/0605003].

[12] N. Arkani-Hamed, A. G. Cohen and H. Georgi, Phys. Rev. Lett. 86, 4757 (2001) [arXiv:hep-th/0104005]; H. C. Cheng, C. T. Hill, S. Pokorski and J. Wang, Phys. Rev. D 64, 065007 (2001) [arXiv:hep-th/0104179].

[13] S. J. Huber and Q. Shafi, Phys. Rev. D 63, 045010 (2001) [arXiv:hep-ph/0005286].

[14] C. Csaki, C. Grojean, L. Pilo and J. Terning, Phys. Rev. Lett. 92, 101802 (2004) [arXiv:hep-ph/0308038]. C. Csaki, C. Grojean, H. Murayama, L. Pilo and J. Terning, Phys. Rev. D 69, 055006 (2004) [arXiv:hep-ph/0305237]. Y. Nomura, JHEP 0311, 050 (2003) [arXiv:hep-ph/0309189]. R. Barbieri, A. Pomarol and R. Rattazzi, Phys. Lett. B 591, 141 (2004) [arXiv:hep-ph/0310285].

[15] M. Golterman and Y. Shamir, Phys. Rev. D 67, 014501 (2003) [arXiv:hep-th/0202162].

[16] P. Hasenfratz, V. Laliena and F. Niedermayer, Phys. Lett. B 427, 125 (1998) [arXiv:hep-lat/9801021]. K. Fujikawa, Nucl. Phys. B 546, 480 (1999) [arXiv:hep-th/9811235].

[17] E. H. Fradkin and S. H. Shenker, Phys. Rev. D 19, 3682 (1979).

[18] J. Giedt, arXiv:hep-lat/0602007. J. Giedt and E. Poppitz, JHEP 0409, 029 (2004) [arXiv:hep-th/0407135]; J. Giedt, R. Koniuk, E. Poppitz and T. Yavin, JHEP 0412, 033 (2004) [arXiv:hep-lat/0410041].

[19] J. Giedt and E. Poppitz, work in progress.

[20] D. Förster, H. B. Nielsen and M. Ninomiya, Phys. Lett. B 94, 135 (1980). M. B. Einhorn and R. Savit, Phys. Rev. D 19, 1198 (1979).

[21] R. Jackiw and R. Rajaraman, Phys. Rev. Lett. 54, 1219 (1985) [Erratum-ibid. 54, 2060 (1985)]. J. Preskill, Annals Phys. 210, 323 (1991). 